\title{
$2 \quad$ Where does digital medicine fit?
}

\section{Within the clinical space}

Clinical care is familiar to most readers from their own experiences with doctors, hospitals and other parts of the healthcare system. Historically, its primary purpose has been to address health problems, and it has long been grounded in the interaction between a patient and a healthcare provider. There has been varying progression of healthcare activities toward preventive care and the maintenance of wellness. With the introduction of connected technologies, there have also been attempts to move healthcare activities into the home, decreasing the need for face-to-face interactions with providers. Clinical care activities include a wide range of diagnostic and treatment processes and procedures, such as:

- real-time monitoring - for example, with continuous blood glucose sensors

- tools to support medical adherence, such as smart apps and pill dispensers

- physical rehabilitation tools, such as digital activity trackers.

Clinical research may include some of the same activities as clinical care, but the primary purpose of clinical research is to develop a better understanding of factors influencing health and illness in people. The federal regulations define research as a 'systematic investigation, including research development, testing and evaluation, designed to develop or contribute to generalizable knowledge' ${ }^{1}{ }^{1}$ When a person (e.g. patient or healthy individual) volunteers to enroll in clinical research, they are called a research participant. There are rules and guidance that must be followed when conducting clinical research to make sure that research participants are protected from undue risks of harm. Clinical research comes in two broad subsets. 
- In interventional studies, participants receive some form of treatment, education or support (Box 2.1). Clinical trials are a subset of interventional studies designed to evaluate the safety and efficacy of an intervention.

- In non-interventional studies, participants do not receive an intervention. Non-interventional studies include observational, exploratory, survey, case-control, cohort and correlational studies. Computational studies that use existing data sources to build predictive models fall into this category.

BOX 2.1

\section{Interventional studies}

In interventional studies, participants are typically randomized at enrollment to either receive the investigational intervention (experimental arm) or the placebo/current standard of care (control arm). Comparing how participants in these two groups respond allows us to understand the safety and efficacy of the intervention.

\section{Outside the clinic walls}

Like any other medical tool, at-home monitoring technologies need to prove their worth. Developers, working with researchers and other experts, must demonstrate that these tools produce clinically meaningful information that leads to clinically meaningful improvements in care, processes and outcomes.

Digital measurement in medicine will not replace clinics or clinicians entirely, nor would we want them to. The delivery of, for example, intravenous drugs or surgery, and the value that patients place on their relationship with their provider, cannot be replaced by digital tools. Nonetheless, when used appropriately, digital measurements can improve care by giving clinicians more complete information. Also, transferring some practices out of the clinic and into patients' regular lives - for example, passively measuring sleep quality with wearables instead of requiring overnight stays in clinics - can enhance access to care and reduce cost. 
Continuous at-home monitoring also raises a new set of practical issues:

- who will monitor the data?

- who will be responsible for acting on it if it indicates a need for action?

- how will providers be compensated for these tasks?

Although organizations like the Clinical Trials Transformation Initiative (CTTI) have made inroads in addressing the first two questions, the field will need to address these issues and adopt consensus solutions for these tools to be truly integrated into clinical care. ${ }^{2}$

A defining moment for any medical product, whether drug or device, is when the product goes to market. From this perspective, the industry splits into 'premarket' research activities, drug and device development in the life sciences and biotech, and 'postmarket' commercial activities, where the products are used in clinical applications like in the hospital. Often, government regulators like the US Food and Drug Administration (FDA) or the Office for Human Research Protections (OHRP) are the gatekeepers between what is considered research (premarket) and what is part of standard of care and commercial activities (postmarket).

National governments are responsible for establishing national medicines and medical device standards and regulatory authorities that determine what claims product manufacturers can make when they go to market in that country. ${ }^{3}$ As of 2015, 121 of the 194 members of the World Health Organization had a national regulatory authority responsible for implementing and enforcing product regulations specific to medical devices. ${ }^{4}$ For example, in the USA, the FDA serves this function. Across the Atlantic, this oversight is provided by the European Medicines Agency (EMA) and in Japan, the Pharmaceuticals and Medical Device Agency (PMDA). 


\section{Key points - where does digital medicine fit?}

- Digital medicine holds the promise of bringing complex medical measurements, observations and interventions outside the clinic.

- Clinical research uses include interventional and non-interventional trials, although many practicalities and data management issues are yet to become commodities.

- Regulation of digital medicine varies widely across the globe.

\section{References}

1. Department of Health and Human Services. Definitions (CFR 46.102). In: Part 46 Protection of Human Subjects; Subpart A - Basic HHS Policy for Protection of Human Research, 2009. www.hhs.gov/ohrp/sites/ default/files/ohrp/policy/ ohrpregulations.pdf, last accessed 31 July 2019.

2. Clinical Trials Transformation Initiative. Recommendations Executive Summary: Advancing the Use of Mobile Technologies for Data Capture \& Improved Clinical Trials, 2018. www.ctti-clinicaltrials.org/sites/ www.ctti-clinicaltrials.org/files/ mobile-technologies-executivesummary.pdf, last accessed 31 July 2019.
3. World Health Organization. Medicines Regulatory Support. www. who.int/medicines/areas/quality_ safety/regulation_legislation/en/, last accessed 31 July 2019.

4. World Health Organization. Global Atlas of Medical Devices. Geneva: World Health Organization, 2017. www.who.int/medical_devices/ publications/global_atlas_meddev 2017/en/, last accessed 31 July 2019. 